\title{
Thoracic surgery experience from the largest pandemic hospital in Europe
}

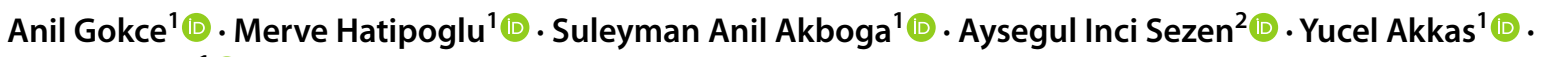 \\ Bulent Kocer $^{1} \mathbb{B}$
}

Received: 11 May 2021 / Accepted: 19 July 2021 / Published online: 31 July 2021

(c) Royal Academy of Medicine in Ireland 2021

\begin{abstract}
Background SARS-CoV-2 coronavirus disease 19 (COVID-19), which was detected in December 2019, whose first cases were observed in Turkey on $11^{\text {th }}$ March 2020, and which was declared a pandemic by the World Health Organization on the same day, has become a public health problem worldwide and has required the adaptation of medical practices to the current situation. In the current paper, we present our experience and practices regarding thoracic surgery from the largest pandemic hospital in Europe over the 1-year period of the pandemic.

Methods Patients who were operated by our thoracic surgery clinic in the largest pandemic hospital in Europe between March 2020 and March 2021 in the COVID-19 pandemic in our country and in the world were evaluated retrospectively.

Results Eighty-five patients were operated on during the 1-year pandemic, of which 54 (63.5\%) were men and 31 (36.5\%) were women. The mean patient age was 47.7 . Morbidity rate was $12 \%$. The average number of PCR tests performed in the preoperative period for COVID-19 disease was 1.6. Sixteen patients had a history of COVID-19 before surgery. COVID-19 was not seen in any patient in our clinic during the postoperative period. Only one patient died out of those who underwent surgery. That patient died due to multiorgan failure.

Conclusion Thoracic surgery has one of the highest risks due to direct contact with the lungs, especially in terms of surgery and the postoperative period. We consider that this risk will be minimized by taking measures during all processes. Moreover, we think that surgical treatments should be delayed as little as possible due to the special status of oncology patients. In addition, considering that if all these rules are followed in the COVID-19 pandemic and in other types of pandemics that may occur in the future, there will be no delay or insufficiency in the treatment of patients and healthcare professionals will be able to work safely.
\end{abstract}

Keywords COVID-19 disease $\cdot$ Experience $\cdot$ Pandemic $\cdot$ Thoracic surgery

\section{Introduction}

SARS-CoV-2 coronavirus disease 19 (COVID-19), which was detected in December 2019, whose first cases were observed in Turkey on $11^{\text {th }}$ March 2020, and which was declared a pandemic by the World Health Organization on the same day, has become a public health problem worldwide and has required

Anil Gokce

anil66gokce@hotmail.com

1 Department of Thoracic Surgery, University of Health Sciences, Ankara City Hospital, Bilkent, Ankara, Turkey

2 Department of Infectious Diseases, Istanbul Bakirkoy Dr. Sadi Konuk Education and Research Hospital, Istanbul, Turkey the adaptation of medical practices to the current situation [1, 2]. Considering the medical and economic difficulties caused by the viral pandemic, it has become necessary to conduct human and resource management. In Turkey, the Ministry of Health decided to discontinue all operations except for emergency surgery on $17^{\text {th }}$ March 2020 [3]. As is known, cancer surgery cannot be evaluated within the scope of emergency surgery, but changes have been required in thoracic surgery [4]. It is not known how long the pandemic will last, and patients due to undergo surgery may lose their chance to have surgery during this period, and in this pandemic, that may cause morbidity and mortality. Delay in surgical treatment option in malignant diseases with changes in time-dependent conditions has been challenging in clinical and ethical terms. During this pandemic, it is important for the continuity of the 
health system that the medical team can work without being affected by the virus [3]. While patients with malignancy having a chance to undergo surgery, it is difficult to apply surgical treatment to patients and healthcare workers without them being affected by the virus. The pandemic has led hospitals to reduce the number of operations and provide human and material resources primarily to COVID-19 patients. While surgical treatment can be delayed in some cancer patients without causing a significant problem, some cancer patients whose treatment is delayed may experience negative effects in terms of survival and quality of life [5]. There are different approaches in many centers regarding the evaluation of patients for surgery, preparation for surgery, the operation, and the postoperative period.

In the current paper, we present our experience and practices regarding thoracic surgery from the largest pandemic hospital in Europe over the 1-year period of the pandemic.

\section{Materials and method}

Ethics committee approval for the study was obtained from the local ethics committee. Patients who were operated by our thoracic surgery clinic in the largest pandemic hospital in Europe between March 2020 and March 2021 in the COVID19 pandemic in our country and in the world were evaluated retrospectively. Eighty-five patients were enrolled in the study. The operated patients were retrospectively evaluated in terms of gender, age, cause of surgery, COVID-19, length of stay, and complications. Thoracotomy, sternotomy, and video-assisted thoracic surgery were used as surgical methods in the patients. The patients were evaluated in terms of the most common complications in thoracic surgery such as infection, bleeding, arrhythmia, embolism, wound infection, and pneumonia. In addition, during the pandemic, the methods applied in terms of protecting both healthcare personnel and patients from COVID-19 were reviewed. In the preoperative period, all relevant examinations were performed in terms of surgical preparation and infection. Consultations were made regarding additional diseases. COVID-19 PCR test was performed at least once preoperatively. In the postoperative period, follow-up and examinations were performed completely in terms of complications and COVID-19 infection. The patients were called for control again 10 days after discharge. Follow-up was also done during this period.

\section{Results}

Ankara City Hospital is the largest hospital in Europe, with a total of 3700 beds, of which 3000 are in wards and 700 are in intensive care. During the pandemic period, the total number of beds was increased to 5500. Due to the number of beds available and the fact that it is a pandemic hospital, it is the largest pandemic hospital in Europe according to the current data. During the study period in our hospital, nearly 30,000 COVID-19 patients were hospitalized and followed up in the service and intensive care units. Our clinic is one of the 3 thoracic surgery clinics in the hospital. During the 1 -year pandemic, nearly 300 patients were operated on in our hospital. In our clinic, 85 patients were operated on during the 1-year pandemic, of which 54 (63.5\%) were men and 31 $(36.5 \%)$ were women (Table 1). The mean patient age was 47.7. COVID-19 was not seen in any patient during the postoperative period. The top 3 most common types of surgery performed in our clinic in a year are lung resection in 26 (30\%) patients, pleural decortication in 13 patients $(15 \%)$, and mediastinal mass excision in $12(14 \%)$ patients. The first 3 symptoms most frequently encountered in patients operated on in our clinic included chest pain in 26 patients, shortness of breath in 9 patients, and cough in 7 patients. Thirty-three patients had no symptoms at the time of admission. Postoperative wound infection was seen in 3 patients, 2 patients had arrhythmia, and 2 patients had bacterial infection. Morbidity rate was $12 \%$. Appropriate antibiotic therapy and frequent dressing were applied for the most common wound infection. Respiratory physiotherapy and appropriate medical treatment were applied for the treatment of pneumonia. For the treatment of arrhythmia and embolism, the patients were consulted with the relevant departments and the recommended treatments were applied completely. The average number of PCR tests performed in the preoperative period for COVID-19 disease was 1.6. Sixteen patients had a history of COVID-19 before surgery. COVID-19 was not seen in any patient in our clinic during the postoperative period. Early PCR tests taken postoperatively in 2 patients who were operated under emergency conditions without testing were positive. This shows that 2 patients have COVID-19 disease during the operation. The early postoperative PCR tests of 2 patients who were taken into emergency surgery due to firearm injuries were positive; these patients were transferred to the pandemic department and followed up after the operation. Both patients were discharged without any problem. In the team involved in these 2 operations, COVID-19 was not seen in any healthcare personnel. We think that our implementation of the precautions mentioned earlier was responsible for this. Only one patient died out of those who underwent surgery. That patient died due to multiorgan failure. We think that effective prevention measures applied by the team are effective in preventing COVID-19 disease in any patient during the postoperative period.

\section{Discussion}

It is thought that the COVID-19 pandemic will show progression like the 1918 influenza pandemic [5]. The treatment timing of oncological diseases directly affects the prognosis of the 
Table 1 Epidemiological characteristics of patients operated by our clinic during the pandemic period

\begin{tabular}{|c|c|c|c|}
\hline & & $\begin{array}{l}\text { Study popula- } \\
\text { tion } \\
N=85\end{array}$ & $\%$ \\
\hline \multicolumn{4}{|l|}{ Gender } \\
\hline & Male & 54 & $63.5 \%$ \\
\hline & Female & 31 & $36.5 \%$ \\
\hline Mean age & 47.7 & & \\
\hline Age range (years) & $16-83$ & & \\
\hline \multicolumn{4}{|l|}{ Symptoms } \\
\hline & Chest pain & 26 & 30 \\
\hline & Cough & 7 & 9 \\
\hline & Asymptomatic & 33 & 38 \\
\hline & Shortness of breath & 9 & 10 \\
\hline & Hemoptysis & 4 & 5 \\
\hline & Difficulty in swallowing & 4 & 5 \\
\hline & Emergency operation & 2 & 3 \\
\hline Preoperative COVID-19 disease & 16 patients & & \\
\hline Postoperative COVID-19 disease & 0 patient & & \\
\hline The average number of PCR tests performed & 1.6 & & \\
\hline Mean length of stay (days) & 9.87 (SD: 5.3) & & \\
\hline \multirow[t]{15}{*}{ Operation types } & Lung resection: & 26 & 30 \\
\hline & Lobectomy & 20 & 15 \\
\hline & Segmentectomy & 4 & 14 \\
\hline & Pneumonectomy & 2 & 9 \\
\hline & Pleural decortication & 13 & 6 \\
\hline & Mediastinal mass excision & 12 & 3 \\
\hline & Bullectomy & 7 & 3 \\
\hline & Hydatic cyst & 5 & 9 \\
\hline & Diaphragmatic rupture & 2 & 3 \\
\hline & Tracheal resection & 2 & 5 \\
\hline & Chest wall resection & 7 & 1 \\
\hline & Esophagectomy & 3 & 2 \\
\hline & Rigid bronchoscopy & 5 & \\
\hline & Rigid esophagoscopy & 1 & \\
\hline & Scoliosis surgery & 2 & \\
\hline \multirow[t]{6}{*}{ Postoperative complications } & No complications & 75 & 89 \\
\hline & Wound infection & 3 & 4 \\
\hline & Arrhythmia & 2 & 2 \\
\hline & Pneumonia & 2 & 2 \\
\hline & Prolonged air leak & 2 & 2 \\
\hline & Emboly & 1 & 1 \\
\hline
\end{tabular}

patients. Surgery performed in the early stages of lung cancer in patients who are followed up and treated with thoracic surgery provides a survival advantage of as high as 90-92\% [6]. Considering all these conditions, delaying the operations of patients with lung cancer who have the chance of surgical treatment may cause the disease to progress. For this reason, the necessity of performing these operations has emerged even during the pandemic period. However, patient selection before trachea and lung operations, where viral load is high, is very important for postoperative morbidity and mortality and for the protection of the surgical team [7]. First of all, it should be ensured that the patients who will be operated do not have COVID-19. Detailed anamnesis of all patients was taken at the time of admission and a clinical examination was performed. A real-time polymerase chain reaction (RTPCR) test was performed twice in the previous week, one of them 3-4 days before the operation, in all patients without suspected contact or symptoms of infection. Considering asymptomatic and presymptomatic patients, it has been stated in the literature that the lungs should be evaluated with computed tomography (CT) 1 day before the operation in terms of parenchymal infiltration [8]. Preoperative tomography was performed in patients undergoing lung resection in our clinic. PCR tests and CT evaluation can be considered negative during the incubation period of 1-14 days in COVID-19 disease $[1,9]$. Therefore, during hospitalization, biochemical parameters such as hemogram, biochemistry, coagulation, and ELISA tests, which are among the preoperative blood tests, 
and C-reactive protein (CRP), ferritin, and D-dimer, which differ in the early stages of COVID-19 disease, were examined in all patients. In the early period of COVID-19 disease, the lymphocyte count may be decreasing, while the leukocyte count may be normal or low [10]. In the pandemic period, the suspicion of COVID-19 disease is very important for the patient and the clinical and surgical team as well as for the selection of patients for surgery. Since the virus is transmitted through droplets and contact, the patients were admitted one to each room. In addition to the informed consent form obtained for all surgical interventions, the patients and their relatives were informed about COVID-19 disease and their consent was obtained before the surgical procedures [11, 12]. These risks should be taken into consideration, especially in patients undergoing trachea and lung surgery. In our clinic, all patients were informed about COVID-19 and their consent was obtained. During the pandemic period, especially minimally invasive (videothoracoscopy [VATS]) procedures should be used in order to reduce patients' long-term hospital stay. With minimally invasive procedures such as VATS performed in lung resections, less pain occurs, and respiratory complications are minimized due to shorter hospital stays compared to thoracotomy [13].

During the pandemic period, it is very important to protect the patient and the surgical team from the risks that occur during surgery and to select the patients in need of urgent surgery carefully. Especially in direct airway operations such as trachea and lung surgery, the possibility of COVID-19 transmission is higher than that in other organ operations. For this reason, the surgery team used an N95 (FFP2/FFP3) mask or double surgical mask and visors during all operations. Especially in patients who have undergone lung surgery, it is necessary to check all areas with the possibility of leakage before the lungs are expanded. Air leakage was checked in all our operations. Close to the end of the operation, a closed-circuit aspiration system was attached to the end of the drain placed in the thorax before the lungs were expanded, and the air in the thorax was aspirated. By using a bacteria filter on the air outlet of the bottle attached to the end of the drain, the possibility of aerosol formation was minimized as a literature [14]. Closed system filtered negative pressure drainage systems were used in lung operations in which leakage could not be checked. In all operations, the surgery team was protected from the amount of aerosol and the leakage of the patients was reduced physiologically $[14,15]$. Similarly, in the literature, it has been reported that exposure to viral load is lower with the HEPA filter double drain bottle technique [16]. Especially in this period, the application of negative pressure and ventilation in all operating rooms will minimize aerosol risk [17]. Considering that all patients going under surgery are possible COVID-19 patients, all healthcare professionals should protect themselves with the recommended equipment. Our operations were performed with as few personnel as possible.
Routine controls and respiratory physiotherapy were performed with protective equipment in the patients who were followed up in single rooms after thoracic surgery. In the postoperative period, the patients' temperature was checked at least four times a day. However, if the fever persists for more than $48 \mathrm{~h}$ despite symptomatic treatment, COVID-19 disease must be ruled out. After excluding causes such as atelectasis, wound infection, and effusion, which may be seen after lung surgery, a nasopharyngeal swab was taken for the diagnosis of COVID-19 and a RT-PCR test was performed. Preoperative laboratory parameters for COVID-19 disease were checked regularly. In particular, secondary to the trauma that occurs after lung surgery, increases in the number of leukocytes and CRP and D-dimer values can be observed. Therefore, it should be kept in mind that monitoring of these parameters in the presence of prolonged fever or additional symptoms is very important in terms of COVID-19 disease. Daily PA lung radiography was performed in all the patients. When pulmonary involvement was suspected, the lungs were evaluated for COVID-19 with chest CT. In order to reduce the possibility of hospital infections in the postoperative period, it was aimed to keep hospital stay as short as possible. Otherwise, the risk of cross infection may be increased [18, 19]. It has been stated in similar publications that videothoracoscopic procedures should be used as much as possible during the pandemic period due to the short hospital stay, especially for thoracic surgery [18-20]. Even if patients hospitalized for a long period ( $>1$ week) with suspected lung involvement did not have symptoms, a RT-PCR test was performed by nasopharyngeal swab for COVID-19 and the lungs were evaluated with control CT. It was ensured that the patients were visited daily by the smallest number of healthcare professionals possible, wearing masks and complying with the distance rules. Respiratory exercises and mobilization of hospitalized patients were provided without their leaving their rooms. In the postoperative period, with these carefully applied methods, no postoperative COVID-19 patients were seen in our clinic. Discharged patients were called for control within the first 10 days and their tests were performed. During this period, COVID-19 disease was not encountered.

\section{Limitation}

The most important limitations of the study are that it is retrospective, the number of patients in the study is small, and it was conducted in a short time period.

\section{Conclusion}

Thoracic surgery has one of the highest risks due to direct contact with the lungs, especially in terms of surgery and the postoperative period. We consider that this risk will be 
minimized by taking measures during all processes. Moreover, we think that surgical treatments should be delayed as little as possible due to the special status of oncology patients. In addition, considering that if all these rules are followed in the COVID-19 pandemic and in other types of pandemics that may occur in the future, there will be no delay or insufficiency in the treatment of patients and healthcare professionals will be able to work safely.

\section{Declarations}

Conflict of interest The authors declare no competing interests.

Meeting presentation This paper has not been presented in any meeting.

\section{References}

1. Lu H, Stratton CW, Tang YW (2020) Outbreak of pneumonia of unknown etiology in Wuhan, China: the mystery and the miracle. J Med Virol 92:401-402. https://doi.org/10.1002/jmv.25678

2. WHO (2020) Timeline of WHO's response to COVID-19 [Online]. https://www.who.int/news-room/detail/29-06-2020covidtimeline. (Accessed 13 Aug 2020)

3. T.C. Sağlık Bakanlı̆̆ 1 Halk Sağ $l_{1} \breve{g ̆}_{1}$ Genel Müdürlüğü, Bilim Kurulu Çalışması (2020) Covid-19 (SARS-CoV-2 Enfeksiyonu) Rehberi.

4. Jheon S, Ahmed AD, Fang VW, Jung W, Khan AZ, Lee JM et al (2020) Thoracic cancer surgery during the COVID-19 pandemic: a consensus statement from the Thoracic Domain of the Asian Society for Cardiovascular and Thoracic Surgery. Asian Cardiovasc Thorac Ann 28(6):322-329. https://doi.org/10.1177/0218492320940162

5. Yun YH, Kim YA, Min YH, Park S, Won YJ, Kim DY et al (2012) The influence of hospital volume and surgical treatment delay on long-term survival after cancer surgery. Ann Oncol 23(10):27312737. https://doi.org/10.1093/annonc/mds101

6. Moon MR, Jones DR, Adams DH, Starnes VA (2020) American Association for Thoracic Surgery: maintaining the mission during the coronavirus disease 2019 (COVID-19) pandemic. J Thorac Cardiovasc Surg 160(3):737-739. https://doi.org/10.1016/j.jtcvs. 2020.05.022

7. Goldstraw P, Chansky K, Crowley J, Rami-Porta R, Asamura H, Eberhardt WEE et al (2016) The IASLC lung cancer staging project: proposals for revision of the TNM stage groupings in the forthcoming (Eight) edition of the TNM classification for lung cancer. J Thorac Oncol 11(1):39-51. https://doi.org/10.1016/j.jtho. 2015.09.009
8. Thoracic Surgery Outcomes Research Network Inc, Antonoff M, Backhus L, Boffa DJ, Broderick SR, Brown LM et al (2020) COVID-19 guidance for triage of operations for thoracic malignancies: a consensus statement from Thoracic Surgery Outcomes Research Network. J Thorac Cardiovasc Surg 160(2):601-605. https://doi.org/10.1016/j.jtcvs.2020.03.0615

9. Huybens EM, Bus MPA, Massaad RA, Wijers L, van der Voet JA, Delfos NM et al (2020) What is the preferred screening tool for COVID-19 in asymptomatic patients undergoing a surgical or diagnostic procedure? World J Surg 13:1-8. https://doi.org/10.1007/ s00268-020-05722-9

10. Tao A, Yang Z, Hou H, Zhan C, Chen C, Lv W et al (2020) Correlation of chest $\mathrm{CT}$ and RT-PCR testing in coronavirus disease 2019 (COVID-19) in China: a report of 1014 cases. Radiology 296(2):E32-E40. https://doi.org/10.1148/radiol.2020200642

11. Rudrapal M, Khairnar SJ, Borse LB, Jadhav AG (2020) Coronavirus disease-2019 (COVID-19): an updated review. Drug Res (Stuttg) 70(9):389-400. https://doi.org/10.1055/a-1217-2397

12. Turnham HL, Dunn M, Hill E, Thornburn GT, Wilkinson D (2020) Consent in the time of COVID-19. J Med Ethics 46(9):565-568. https://doi.org/10.1136/medethics-2020-106402

13. Federation of Surgical Specialty Associations (2020) Developing Safe Surgical Services (DSSS) for the Covid19. Era. Available at:https:// fssa.org.uk/_userfiles/pages/files/covid19/developing_safe_surgical_ services_dsss_for_the_covid19_era_may2020updated.pdf. (Accessed 11 Dec 2020)

14. Sihoe AD, Yim AP (2008) Video-assisted pulmonary resections. In: Patterson FG, Cooper JD, Desla-uriers J (eds) Thoracic Surgery, 3rd edn. Elsevier, Philadelphia, pp 970-988

15. Shen C, Jiang L, Ma H, Che G (2020) Perioperative preparation in thoracic day surgery: battle against COVID-19. Thorac Cancer 11(8):2376-2379. https://doi.org/10.1111/1759-7714.13500

16. Mariani AW, Pêgo-Fernandes PM (2020) Thoracic surgery in a hospital dedicated to treating COVID-19: challenges and solutions. Clinics (Sao Paulo) 75:e1982. https://doi.org/10.6061/clinics/2020/e1982

17. Ceylan KC, Batihan G, Yazgan S, Gursoy S, Kıraklı SC, Ataman S (2020) Pleural complications in patients with coronavirus disease 2019 (COVID-19): how to safely apply and follow-up with a chest tube during the pandemic? Eur J Cardiothorac Surg 58(6):12161221. https://doi.org/10.1093/ejcts/ezaa356

18. Ti LK, Ang LS, Foong TW, Ng BSW (2020) What we do when a COVID-19 patient needs an operation: operating room preparation and guidance. Can J Anaesth 67(6):756-758. https://doi.org/10. 1007/s12630-020-01617-4

19. Li X, Liu M, Zhao Q, Liu R, Zhang H, Dong M et al (2020) Preliminary recommendations for lung surgery during COVID-19 epidemic period. Thorac Cancer 11(6):1372-1374. https://doi.org/10. 1111/1759-7714.13423

20. Muñoz-Largacha JA, Wei B (2020) Commentary: lung surgery in the time of COVID-19. J Thorac Cardiovasc Surg 160(2):e97-e98. https://doi.org/10.1016/j.jtcvs.2020.04.088 